\title{
Late seroconversion in HIV-resistant Nairobi prostitutes despite pre-existing HIV-specific $\mathrm{CD8}^{+}$responses
}

\author{
Rupert Kaul,, ${ }^{1,2}$ Sarah L. Rowland-Jones, ${ }^{2}$ Joshua Kimani, ${ }^{1}$ Tao Dong, ${ }^{2}$ Hong-Bing Yang, ${ }^{2}$ \\ Peter Kiama, ${ }^{1}$ Timothy Rostron, ${ }^{2}$ Ephantus Njagi, ${ }^{1}$ Job J. Bwayo, ${ }^{1}$ Kelly S. MacDonald, ${ }^{3}$ \\ Andrew J. McMichael, ${ }^{2}$ and Francis A. Plummer ${ }^{1,4}$
}

\author{
${ }^{1}$ Department of Medical Microbiology, University of Nairobi, Nairobi, Kenya \\ ${ }^{2}$ Human Immunology Unit, Institute of Molecular Medicine, John Radcliffe Hospital, Oxford, United Kingdom \\ ${ }^{3}$ Division of Infectious Diseases, Department of Medicine, University of Toronto, Toronto, Canada \\ ${ }^{4}$ Department of Medical Microbiology, University of Manitoba, Winnipeg, Canada
}

Address correspondence to: Rupert Kaul, Medical Research Council Human Immunology Unit, Institute of Molecular Medicine, Molecular Immunology Group, John Radcliffe Hospital, Headington, Oxford OX3 9DS, United Kingdom. Phone: 01865-222312; Fax: 01865-222502; E-mail: rupertkaul@hotmail.com.

This work was presented in part at the 7th Conference on Retroviruses and Opportunistic Infections, in San Francisco, California, USA, January 29-February 2, 2000.

Received for publication July 6, 2000, and accepted in revised form December 10, 2000.

\begin{abstract}
Resistance to HIV infection in a small group of Kenyan sex workers is associated with CD8 ${ }^{+}-1 \mathrm{ym}$ phocyte responses to HIV cytotoxic T-lymphocyte (CTL) epitopes. Eleven prostitutes meeting criteria for HIV resistance seroconverted between 1996 and 1999. The occurrence and specificity of preexisting HIV-1 epitope-specific responses were examined using the IFN- $\gamma$ enzyme-linked immunospot assay, and any epitopes recognized were cloned and sequenced from the infecting viral isolate. Immunologic and behavioral variables were compared between late seroconverters and persistently uninfected sex worker controls. HIV-1 CTL epitope responses were present in four of six cases, 5-18 months before seroconversion, and their presence was confirmed by bulk CTL culture. A possible viral escape mutation was found in one of six epitopes. The key epidemiologic correlate of late seroconversion was a reduction in sex work over the preceding year. In persistently uninfected controls, a break from sex work was associated with a loss of HIV-specific $\mathrm{CD}^{+}{ }^{+}$responses. Late seroconversion may occur in HIV-1-resistant sex workers despite preceding HIV-specific $\mathrm{CD}^{+}$ responses. Seroconversion generally occurs in the absence of detectable CTL escape mutations and may relate to the waning of $\mathrm{HIV}$-specific $\mathrm{CD8}^{+}$responses due to reduced antigenic exposure.
\end{abstract}

J. Clin. Invest. 107:341-349 (2001).

\section{Introduction}

Individuals exhibit different susceptibility to infection by HIV-1, with a minority of individuals remaining uninfected despite repeated viral exposure (1-3). HIV1 -specific immune responses, in particular cytotoxic $T$ lymphocytes (CTL), may play an important role in protection from infection in HIV-1-exposed, persistently seronegative (HEPS) subjects (1-3). The generation of HIV-1 CTL responses has therefore been identified as a key goal in the effort to develop a preventative HIV-1 vaccine (4). However, the durability and protective efficacy of CTL responses in HEPS subjects is unclear.

HIV-1-specific CTL may play an important role in controlling viremia (1). High levels of HIV-1-specific CTL are found in acute infection and may fall below the limits of detection as the early peak in viral load decreases, particularly in the context of complete viral suppression with antiretroviral therapy (5-7). In chronic infection there is an inverse correlation between the numbers of circulating HIV-specific $\mathrm{CD} 8^{+} \mathrm{T}$ cells and the plasma viral load (8), but this population also declines to undetectable levels after successful antiretroviral therapy $(5,9,10)$. These observations suggest that the maintenance of a circulating HIV-1-specific effector CTL population, which directly kills infected cells and produces antiviral cytokines such as IFN- $\gamma$, is dependent on an ongoing antigenic stimulus. However, a memory CTL population, which can undergo multiple divisions and replenish the effector population (11), is maintained in HIV-1-infected subjects after antiretroviral therapy has reduced viremia below the limits of detection (6).

Transient HIV-1 exposure in seronegative individuals may result in the generation of HIV-1-specific CTL (1-3), but whether this exposure results in long-lived HIV-1-specific CTL memory is unknown. CTL responses can be detected after a single percutaneous exposure to infected blood, but fall below the limits of detection within 2-8 months (12). Most studies of HIV-specific CTL after transient exposure (12-18) have studied CTL activity by in vitro culture of PBMCs after restimulation with HIV antigens, which is pre- 
sumed to expand both memory and effector CTL populations (19). The loss of these responses within months of viral exposure therefore suggests a loss of both effector and memory CTL in the periphery. This is in keeping with other virus infections, where maintenance of protective memory CTL in the periphery is short-lived in the absence of antigen (20).

The waning of protective CTL immunity in the absence of antigen therefore constitutes a potential problem confronting a CTL-based HIV vaccine. A second theoretical problem is the evasion of protective epitope-specific CTL by viral variants bearing amino acid substitutions within the target epitope, as has been seen in a vaccine model of simian immunodeficiency virus (SIV) infection (21). The phenomenon of CTL "escape" is well described in HIV-1-infected subjects, where it may be associated with increased viral load and disease progression (22-24).

A small group of sex workers in Nairobi, Kenya, has remained uninfected by HIV-1 despite intense exposure and are defined as resistant to HIV-1 infection (25). HIV-1 resistance in this cohort is not due to altered cellular susceptibility to HIV-1 or to known chemokine-receptor polymorphisms (26), but has been associated with certain HLA class I and II alleles (27), $\mathrm{CD}^{+}$responses to HIV-1 CTL epitopes $(18,28)$, T-helper responses (29), and HIV-1-specific IgA (29). In the 4-year period from 1996 to 2000, eleven women who had met criteria for HIV-1 resistance seroconverted. We have examined these late seroconverters for evidence of preceding HIV-1-specific $\mathrm{CD}^{+}$responses and have explored the possibility that late seroconversion may represent infection by primary viral escape variants or waning of epitope-specific immunity.

\section{Methods}

Study populations and general laboratory methods. Women were enrolled through a dedicated sex worker clinic in Nairobi, Kenya $(30,31)$ and were classified as HIV1-resistant if they remained HIV-1 seronegative and PCR negative for at least 3 years while continuing in sex work (25). Late seroconverters (cases) comprised women who had met criteria for HIV-1 resistance, but subsequently seroconverted between January 1996 and December 1999. Each case was compared with two persistently seronegative sex workers (controls) who: (a) had been reviewed in the clinic in the same year as the index case; (b) had at least one PBMC sample available; and (c) had been enrolled in the cohort at as close a time as possible to the index case to control for changes in sexual behavior over time. Lower-risk HIV-1 uninfected control women were enrolled from a familyplanning clinic in Nairobi that is participating in ongoing HIV-1 surveillance. Informed consent was obtained from all study participants, and the study conformed to ethical guidelines from the University of Manitoba and the University of Nairobi.

HIV-1 serological testing employed a synthetic peptide enzyme immunoassay (Detect HIV; Biochem
ImmunoSystems Inc., Montreal, Quebec, Canada), and positive tests were confirmed using a recombinant antigen enzyme immunoassay (Recombigen HIV-1/2 EIA; Cambridge Biotech Corp., Galway, Ireland). All HIV1-seronegative sex workers were confirmed to be HIV-1 uninfected by employing a PCR system that uses primers for env, nef, and vif HIV-1 provirus genes specifically adapted to detect African clades and that is sensitive to below five viral copies per $2 \times 10^{5} \mathrm{PBMC}(32)$. Molecular HLA typing was performed on all study subjects using amplification refractory mutation system PCR with sequence-specific primers, as described previously (33). Peripheral blood T-lymphocyte subset analysis was performed using anti-CD4 FITC/CD8 phycoerythrin (PE; Becton-Dickinson Immunocytometry Systems, San Jose, California, USA).

HIV-1 epitope selection and peptide synthesis. HIV-1 peptides were selected from a panel of previously defined A-, B-, and D-clade CTL epitopes based on the class I HLA haplotype of the donor. Peptides were synthesized by F-moc chemistry using a Zinnser Analytical synthesizer (Advanced Chemtech Inc., Louisville, Kentucky, USA), and purity was established by high-pressure liquid chromatography (HPLC).

Peptide-based IFN- $\gamma$ ELISPOT assays. A modified ELISPOT assay was used to detect epitope-specific IFN- $\gamma$ release by either freshly separated or cryopreserved PBMCs, as previously described (28). Ninety-six-well nitrocellulose plates were precoated with a first layer IFN- $\gamma \mathrm{mAb}$, 1-DIK (MABTECH AB, Nacka, Sweden). At least $10^{5}$ PBMCs $\left(2 \times 10^{5}\right.$ cells with a 1:2 dilution where cell numbers allowed) were added to duplicate wells, either with predefined HIV-1 class I-restricted peptide epitopes at a concentration of $20 \mu \mathrm{M}$, with media alone (negative control), or with 1:100 phytohemagglutinin (PHA positive control; Murex Biotech Ltd., Dartford, United Kingdom; ). Plates were incubated overnight at $37^{\circ} \mathrm{C}$ in $5 \% \mathrm{CO}_{2}$, the cells discarded, and the plate incubated at room temperature for 3 hours with a second biotinylated anti-IFN- $\gamma$ monoclonal (7-B6-1 biotin; MABTECH AB), followed by streptavidin-conjugated alkaline phosphatase (MABTECH $\mathrm{AB})$ for 2 hours. Individual IFN- $\boldsymbol{\gamma}$-producing cells were detected as dark-blue spots using an alkaline phosphatase-conjugate substrate kit (Bio-Rad Laboratories Inc., Hercules, California, USA). Spots were counted by using a dissecting microscope $(\times 40)$, and positive results were confirmed using an automated ELISPOT reader (Autoimmun Diagnostika GmbH, Strassberg, Germany). Criteria for a positive HIV-1-specific ELISPOT assay. HIV1 -specific IFN- $\gamma$ responses were reported as the number of spot-forming units (SFU) per $10^{6}$ mononuclear cells, after subtraction of background IFN- $\gamma$ secretion. An HIV-1-specific ELISPOT response was defined previously (28) as: (a) IFN- $\gamma$ release seen in response to 1:100 PHA; (b) greater than or equal to $20 \mathrm{HIV}-1$-specific SFU/10 mononuclear cells; (c) SFU in HIV-1 peptide wells exceeded background by a factor of at least 2; and (d) where serial dilutions had been established, a titratable response was required. 
CTL bulk cultures. Peptide-stimulated CTL bulk assays were performed as described previously (18). Cells were pelleted and pulsed for 1 hour with $100 \mu \mathrm{M}$ of synthetic peptides selected on the basis of HLA haplotype, then suspended in RPMI with 10\% FCS (R10) and cultured at 2 million cells per well in a 24-well Costar plate. Recombinant IL-7 was added to a final concentration of $25 \mathrm{ng} / \mathrm{ml}$ on day 1, and IL-2 (Lymphocult-T; Biotest, Solihull, United Kingdom) was added to a dilution of $10 \%$ on day 3. After 10-14 days in culture, assays for HIV-1-specific CTL activity were carried out using class I-matched B-lymphoblastoid target cells pulsed with the appropriate peptides in a $\mathrm{Cr}^{51}$ release assay. Lysis of peptide-pulsed targets was considered to be HIV-1 specific if lysis was at least $10 \%$ more than that of the unpulsed control.

HIV-1 epitope sequencing. Two to four million postseroconversion PBMCs were incubated in R10 with 1:100 PHA (Murex Biotech Ltd.) for 36-48 hours. All PBMC samples were obtained within 6 months of seroconversion. DNA was extracted using the Purgene system (Gentra Systems Inc., Minneapolis, Minnesota, USA). CTL epitopes recognized by donor PBMCs prior to seroconversion were amplified using nested PCR with Expand High Fidelity Taq (Roche Molecular Biochemicals, Mannheim, Germany), as described previously (24). DNA fragment size was confirmed using a PhiX 174 Hae ladder (New England Biolabs Inc., Beverly, Massachusetts, USA), and DNA was purified by passage over Sephadex G-50 beads (Sigma Chemical Co., St. Louis, Missouri, USA). DNA insertion into the plasmid PCR4-TOPO and transformation of Escherichia coli were performed using the TOPO-TA system (Invitrogen, Gronigen, The Netherlands), according to the manufacturer's instructions. Bacteria were grown overnight at $37^{\circ} \mathrm{C}$ on an LB ampicillin plate coated with $40 \mu \mathrm{l}$ of $40 \mathrm{mg} / \mathrm{ml} \mathrm{X}$-gal in dimethyl formamide (DMF). Twenty transformed colonies were selected, cultured overnight in LB ampicillin medium, and plasmid DNA isolated using the QIAprep Spin Miniprep Kit (QIAGEN, Crawley, United Kingdom). DNA sequencing was performed using the Beckman Coulter CEQ-200 (Beckman Coulter Inc., Miami, Florida, USA).

Case-control study. Behavioral data up to the time of seroconversion was obtained from standardized questionnaires administered at recruitment to the clinic and annually thereafter (31). Data collected include the number of daily clients, consistency of condom use, methods of contraception, prior duration of prostitution, sexual practices with

Table 1 clients, and other HIV-1 risk factors such as blood transfusion and intravenous drug use. Additional information obtained at the time of seroconversion included any break from sex work over the past year, any recent medical events such as vaccination or any new medication(s), and whether the subject had been raped during the past year or had had known contact with an HIV-1-infected partner.

Data analysis. Statistical analysis used the SPSS for Windows Rel. 9.0.0 1998 package (SPSS Inc., Chicago, Illinois, USA). Dichotomous variables were compared between groups using the Mantel-Haenszel common odds ratio estimate. Comparison of means between study groups was performed by ANOVA. Kaplan-Meier survival analysis was performed to examine the probability of seroconversion as a function of time.

\section{Results}

Preceding HIV-1 epitope-specific responses in seroconverters. Late seroconversion was documented over the study period in eleven of 114 (9.6\%) sex workers meeting criteria for HIV-1 resistance (25). Preseroconversion PBMC specimens had been drawn in 7 of 11 cases, and predefined HLA class I CTL epitope peptides were available for six of seven cases. HIV-1-specific responses were found at the last time tested before seroconversion in four of six (67\%) cases, 5-18 months before HIV-1 seroconversion (Table 1), with response frequencies from 20-168 SFU/106 PBMCs. In cases ML 857 and ML 1203, epitope-specific responses were detected at greater than or equal to two time points before seroconversion and were confirmed using a bulk CTL chromium-release assay (including the low-frequency ELISPOT response in ML 1203 on January 3, 1997, to epitope DTVLEDINL at $20 \mathrm{SFU} / 10^{6} \mathrm{PBMCs}$ ). Cases where preseroconversion PBMCs were available did not differ from cases without PBMCs in HIV-exposure parameters

HIV-1 CTL epitope-specific assays run before seroconversion in six cases

\begin{tabular}{|c|c|c|c|c|}
\hline $\begin{array}{l}\text { Subject } \\
\text { (conversion date) }\end{array}$ & Assay date & $\begin{array}{l}\text { Peptide epitope } \\
\text { response }\end{array}$ & $\begin{array}{l}\text { HLA restriction, HIV } \\
\text { gene product (clade) }\end{array}$ & $\begin{array}{l}\text { SFU } / 10 \\
\text { PBMCs }\end{array}$ \\
\hline $\begin{array}{l}\text { ML 857 } \\
(08 / 28 / 98)\end{array}$ & $\begin{array}{l}03 / 01 / 97 \\
10 / 21 / 97 \\
06 / 09 / 98\end{array}$ & $\begin{array}{c}\text { DTVLEDINL } \\
\text { H/NPDIVIYQY } \\
\text { DTVLEDINL } \\
\text { DTVLEDINL } \\
\text { H/NPDIVIYQY }\end{array}$ & $\begin{array}{c}\mathrm{A}^{*} 6802 \mathrm{pol}(\mathrm{A}) \\
\mathrm{B} 35 \mathrm{pol}(\mathrm{A} / \mathrm{B}) \\
\mathrm{A}^{*} 6802 \mathrm{pol}(\mathrm{A}) \\
\mathrm{A}^{*} 6802 \mathrm{pol}(\mathrm{A}) \\
\mathrm{B} 35 \mathrm{pol}(\mathrm{A} / \mathrm{B})\end{array}$ & $\begin{array}{c}100^{\mathrm{A}} \\
100^{\mathrm{A}} \\
65 \\
113 \\
75\end{array}$ \\
\hline $\begin{array}{l}\text { ML 1203 } \\
(06 / 04 / 98)\end{array}$ & $\begin{array}{l}03 / 01 / 97 \\
12 / 17 / 97\end{array}$ & $\begin{array}{l}\text { DTVLEDINL } \\
\text { FPVTPQVPLR } \\
\text { DTVLEDINL } \\
\text { FPVTPQVPLR }\end{array}$ & $\begin{array}{c}A^{*} 6802 \text { pol } \\
\text { B7 nef(B) } \\
A^{*} 6802 \text { pol (A) } \\
\text { B7 nef (B) }\end{array}$ & $\begin{array}{l}20^{\mathrm{A}} \\
\mathrm{nd}^{\mathrm{A}} \\
168 \\
43\end{array}$ \\
\hline $\begin{array}{l}\text { ML } 1575 \\
(05 / 08 / 98)\end{array}$ & $\begin{array}{l}06 / 10 / 97 \\
10 / 21 / 97\end{array}$ & $\begin{array}{l}\text { none } \\
\text { none }\end{array}$ & & \\
\hline $\begin{array}{l}\text { ML } 1592 \\
(07 / 07 / 96)\end{array}$ & $04 / 18 / 96$ & none & & \\
\hline $\begin{array}{l}\text { ML } 1707 \\
(01 / 05 / 98)\end{array}$ & $10 / 23 / 97$ & DTVLEDINL & $A^{*} 6802 \mathrm{pol}(\mathrm{A})$ & 36 \\
\hline $\begin{array}{l}\text { ML 1760 } \\
(05 / 16 / 98)\end{array}$ & $05 / 30 / 97$ & ILKD/EPVHGV & A2 pol (A/B) & 90 \\
\hline
\end{tabular}

See text for definition of an HIV-1 epitope-specific response. AThese ELISPOT results were confirmed using bulk cytotoxic T-lymphocyte assay (see text for assay methods). 
Table 2

Amino acid sequences of HIV-1 CTL epitopes recognized in preseroconversion ELISPOT assays and sequence of infecting virus

\begin{tabular}{ccc} 
Subject & $\begin{array}{c}\text { Epitope sequence: } \\
\text { preseroconversion } \\
\text { ELISPOT }\end{array}$ & $\begin{array}{c}\text { Epitope sequence: } \\
\text { infecting virus (proportion } \\
\text { of isolates sequenced) }\end{array}$ \\
$M L 857$ & DTVLEDINL & DTVLEDINL $(20 / 20)$ \\
$M L 1203$ & HPDIVIYQY & NPQIIIYQY $(20 / 20)$ \\
& DTVLEDINL & DTVLEDINL $(20 / 20)$ \\
$M L 1707$ & FPVTPQVPLR & FPVTPQVPLR $(20 / 20)$ \\
$M L 1760$ & DTVLEDINL & DTVLEDINL $(20 / 20)$ \\
\hline
\end{tabular}

See text for definition of an HIV-1 epitope-specific response.

over the year preceding seroconversion, either in the duration of previous seronegative follow-up (7.7 vs. 6.2 years; $P=0.2$ ), the number of clients per day (3.8 vs. 3.2; $P=0.7)$, or condom use (78\% vs. $55 \% ; P=0.4)$. No samples drawn from seroconverters within 2 months before their presumed date of infection were tested.

The most common HIV-1 CTL epitope recognized was DTVLEDINL, an A-clade, A*6802-restricted HIV-1 pol epitope ( $n=3$ : ML 857, ML 1203, ML 1707). Other epitopes recognized included ILKDPVHGV, an A-clade, A2-restricted pol epitope ( $n=1$ : ML 1760); FPVTPQVPLR, a B7-restricted nef epitope $(n=1$ : ML 1203); and HPDIVIYQY, a B35-restricted, A-clade pol epitope ( $n=1$ : ML 857).

Control assays were run at a single time point for 18 lower-risk, seronegative Kenyan women, and all were negative. The mean number of epitopes screened in lower-risk women was 5.9; epitope DTVLEDINL was tested in six women; epitope ILKDPVHGV in two; epitope HPDIVIYQY in three; and epitope FPVTPQVPLR in one woman.

DNA sequencing of HIV-1 CTL epitopes. A total of six HIV-1 CTL epitopes were recognized in preseroconversion ELISPOT assays from four women and were amplified and sequenced from the infecting viral strains (Table 2). Twenty epitope sequences were obtained in each case to rule out sequence variation in rare quasispecies. In five of six viral epitopes, the infecting sequence was identical (20/20 sequences) to that recognized in preseroconversion ELISPOT and/or CTL assays (see Table 1). However, the sequence of the B35-restricted HIV-1 pol epitope in seroconverter ML 857 differed from that recognized preseroconversion (NPEIIIYQY vs. HPDIVIYQY; amino acid substitutions shown in bold), although these amino acid substitutions have all been described previously in clade $\mathrm{A}$ HIV-1 strains (34).

Potential CTL escape variant detected in donor ML 857. Bulk CTL assays were performed for ML 857 using preseroconversion PBMCs stimulated by either the HPDIVIYQY or NPEIIIYQY epitope variants (Figure 1). The HPDIVIYQY-stimulated CTL line demonstrated lowlevel specific lysis of targets pulsed with epitope HPDIVIYQY (as recognized in the ELISPOT assay), but not with epitope NPEIIIYQY. The NPEIIIYQY-stimulated CTL line did not recognize targets pulsed with either epitope. This confirms that preseroconversion PBMCs from this HEPS subject recognized epitope HPDIVIYQY, but not the infecting variant NPEIIIYQY.

Case-control analysis. Demographics and HIV-1 risktaking behavior did not differ significantly between the 11 seroconverters and 22 matched, persistently seronegative controls (Table 3 ). Risk factors such as anal sex, sex during menses, and intravenous drug use were rare, and no subject reported nonconsensual sex during the past year, recent vaccination, or recent blood transfusion. There was no association between seroconversion and method of contraception or the occurrence of a sexually transmitted infection (STI) over the past year. Having stopped sex work entirely for at least 2 months during the preceding year was associated with seroconversion ( $82 \%$ of seroconverters vs. $41 \%$ of controls; odds ratio [OR] $=6.5,95 \%$ confidence interval $[\mathrm{CI}], 1.1-37.5 ; P=0.04)$, as was a reduction in the number of daily clients by two or more per day (55\% vs. $18 \%$ OR $=5.4,95 \% \mathrm{CI}, 1.1-26.9 ; P=0.04)$. Overall reduction in sex work, defined as either having taken a break or having reduced the number of daily clients, was therefore also significantly associated with late
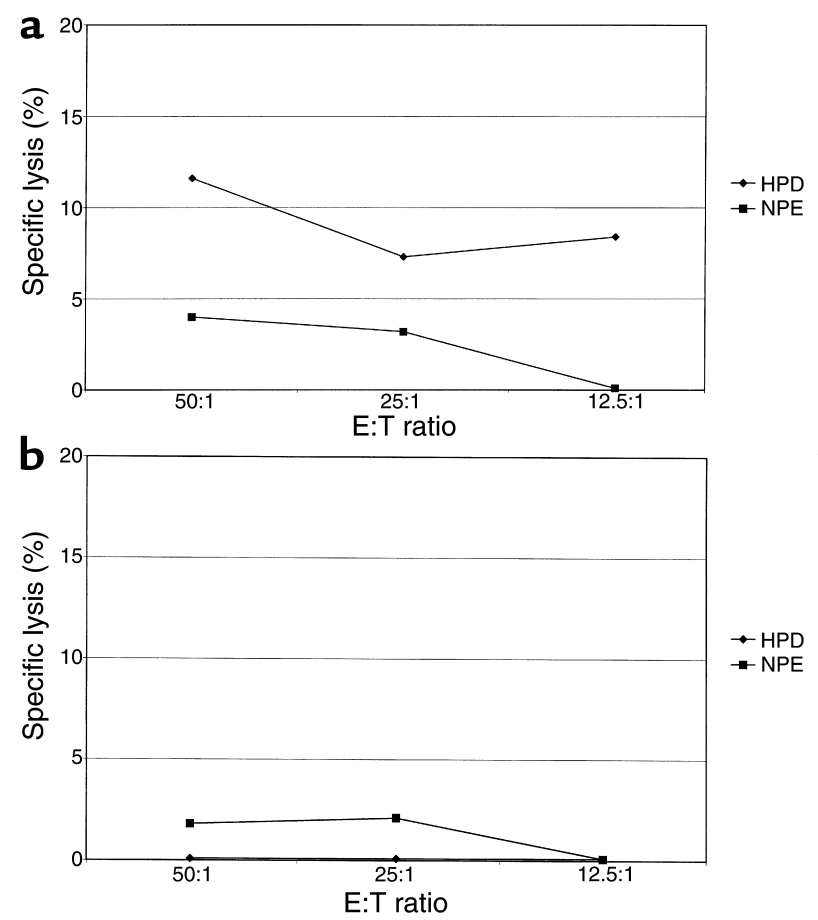

\section{Figure 1}

Effect of epitope variation on recognition of peptide-pulsed targets by ML 857, using PBMCs obtained before seroconversion. (a) HPDIVIYQY-stimulated bulk CTL line recognizes autologous B lymphoblastoid cell line (BCL) targets pulsed with peptide HPDIVIYQY, but not infecting variant NPQIIIYQY. (b) NPEIIIYQY-stimulated bulk $C T L$ line does not recognize autologous $B C L$ targets pulsed with either peptide HPDIVIYQY or NPEIIIYQY. Recognition is defined as greater than $10 \%$ specific lysis of peptide-pulsed target cells by a bulk CTL line (see text for assay details). 
seroconversion $(91 \%$ vs. $45 \%$; OR $=12.0,95 \% \mathrm{CI}$, $1.3-110.5 ; P=0.03)$. Seroconverters who had stopped sex work temporarily had done so for an average of 12.4 months (range 2-60 months), and there was no significant difference in "break" duration between sex workers who seroconverted and persistently seronegative controls (12.4 vs. 5.7 months; $P=0.4$ ).

Reasons for temporarily stopping sex work were most commonly social (traveling home, other employment, social commitments). Of the 9 of 11 cases who had taken a break from sex work, four reported no sexual activity over this time, and one reported sex with a single partner who tested HIV-1 seronegative. The remaining four women reported monogamy or serial monogamy with partner(s) of unknown serostatus and who were unwilling to present for HIV-1 testing. No late seroconverter had stopped sex work due to illness, although four of 11 women described flulike symptoms upon resumption of sex work, which could have been compatible with acute HIV-1 infection.

Late seroconversion was seen in two women who had not taken a break from sex work, ML 1575 and ML 1760. An HIV-1-specific response had been detected 1 year before seroconversion in ML 1760, but no responses had been found in ML 1575 (Table 1). While ML 1760 had not reduced the number of daily clients $(n=5$ clients per day), condom use had increased from $86 \%$ to $100 \%$ over the year preceding seroconversion and then fallen again to $80 \%$ at the time of seroconversion.

HIV-1 epitope responses in persistently seronegative controls. Seroconverter samples from the appropriate time points were not available to demonstrate that stopping sex work had been followed by a decline in epitope-specific responses with subsequent seroconversion. We therefore elected to study HIV-1 epitope-specific responses prospectively in the 22 persistently HIV-1-seronegative sex worker controls who did or did not report a period of reduced sex work. HLA class I epitopes were not available for ML 1705. No HIV-1-specific ELISPOT responses were found at any time for 7 of 21 controls (33\%), while responses were demonstrated at one or more (range 1-5) time points for 14 of 21 controls (68\%; Table 4).

Seven ELISPOT-positive controls (ML 851, ML 1192, ML 1250, ML 1437, ML 1693, ML 1732, and ML 1749) reported a break from sex work of 2 months or more during the study. In six of seven cases, HIV-1-specific responses were no longer seen in PBMCs drawn 2-8 months after this break. Retirement from sex work was associated with the loss of HIV-1-specific responses in three of four cases (ML 1192 and ML 851 shown in Figure 2 , a and $b$ ), although one of four

\section{Table 3}

cases maintained a response to the B18 224 epitope FRDYVDRFFK for more than 1 year (ML 1732; Figure 2c). The HIV-1 serostatus of her regular sex partner was unknown. ML 1437 reported two breaks from sex work and lost an initial response to $A^{*} 6802$ pol epitope DTVLEDINL (Figure 2d). Three women resumed sex work after a break (ML 1250, ML 1749, and ML 851, who later retired completely), and in each case HIV-1-specific responses were redetected after a lag of 1-12 months (Figure 2, e, f, and b). In ML 1250 a broad response was detected after restarting sex work, but waned 8 months later without any change in risk behavior.

HIV-1-specific responses were detected in seven persistently seronegative controls who had not taken a break from sex work. PBMCs were only available for ML 1573 at a single time point so that serial assays could be performed in six women. In four cases (ML 887, ML 1601, ML 1671, ML 1792) HIV-1-specific responses could be detected throughout the study. The specificity of these responses was constant in two controls (ML 1601, $\mathrm{A}^{*} 6802$ pol epitope DTVLEDINL and ML 1671, $\mathrm{A}^{*} 6802$ RT epitope ETAYFILKL; Figure 2g) and varied in two women (in ML 1792 from B14 restricted $p 24$ epitope DLNMMLNIV to RAEQASQEV; in ML 887 as shown in Figure 2h). In ML 1589 an initial response was lost over the course of the study without a reported break from sex work or change in risk behavior. ML 1668 had no initial response, but developed responses to A33 pol epitope AIFQSSMTK and B49 nef epitope YPLTFGWCY/F after 9 months, while reducing condom use from $100 \%$ to $33 \%$.

There was a significant overall association between stopping sex work and a loss of HIV-1 epitope-specific responses: six of seven women who lost responses

Case-control comparison of late seroconverters with enrollment-matched controls remaining HIV-1 seronegative

\begin{tabular}{|c|c|c|c|}
\hline & $\begin{array}{l}\text { Seroconverters } \\
\quad(n=11)\end{array}$ & $\begin{array}{c}\text { Seronegative } \\
\text { controls }(n=22)\end{array}$ & $\begin{array}{c}P \text { value }^{\mathrm{A}} \\
(\mathrm{OR}, 95 \% \mathrm{Cl})\end{array}$ \\
\hline Mean age (years) & 35.3 & 37.8 & 0.3 \\
\hline Duration of follow-up (years) & 6.9 & 6.8 & 0.9 \\
\hline Prostitution duration (years) & 10.8 & 12.0 & 0.6 \\
\hline Condom use at enrollment (\%) & 73.5 & 70.0 & 0.8 \\
\hline Condom use at seroconversion (\%) & 80 & 82.4 & 0.7 \\
\hline Clients/day at enrollment & 5.5 & 6.1 & 0.6 \\
\hline Clients/day at seroconversion & 2.4 & 3.7 & 0.3 \\
\hline $\begin{array}{l}\geq 2 \text {-month break from sex work } \\
\text { in past year }\end{array}$ & $9 / 11$ & $9 / 22$ & $\begin{array}{c}0.04 \\
(6.5 ; 1.1-37.5)\end{array}$ \\
\hline Fewer clients in past year ${ }^{B}$ & $6 / 11$ & $4 / 22$ & $\begin{array}{c}0.04 \\
(5.4 ; 1.1-26.9)\end{array}$ \\
\hline Either break or fewer clients & $10 / 11$ & $10 / 22$ & $\begin{array}{c}0.03 \\
(12.0 ; 1.3-110.5)\end{array}$ \\
\hline New high-risk sexual behavior ${ }^{C}$ & $4 / 11$ & $3 / 22$ & $\begin{array}{c}0.2 \\
(3.6 ; 0.6-20.4)\end{array}$ \\
\hline Injection drug use, past year & $1 / 11$ & $0 / 22$ & $\begin{array}{c}0.15 \\
(\infty ; N A)\end{array}$ \\
\hline
\end{tabular}

AANOVA for comparison of means; Mantel-Haenszel common odds ratio estimate for comparison of discrete variables. ${ }^{B}$ Reduction in number of daily clients by two or more over the past year. ${ }^{\mathrm{C}}$ Either started to practice anal intercourse or sex during menses over the past year. 

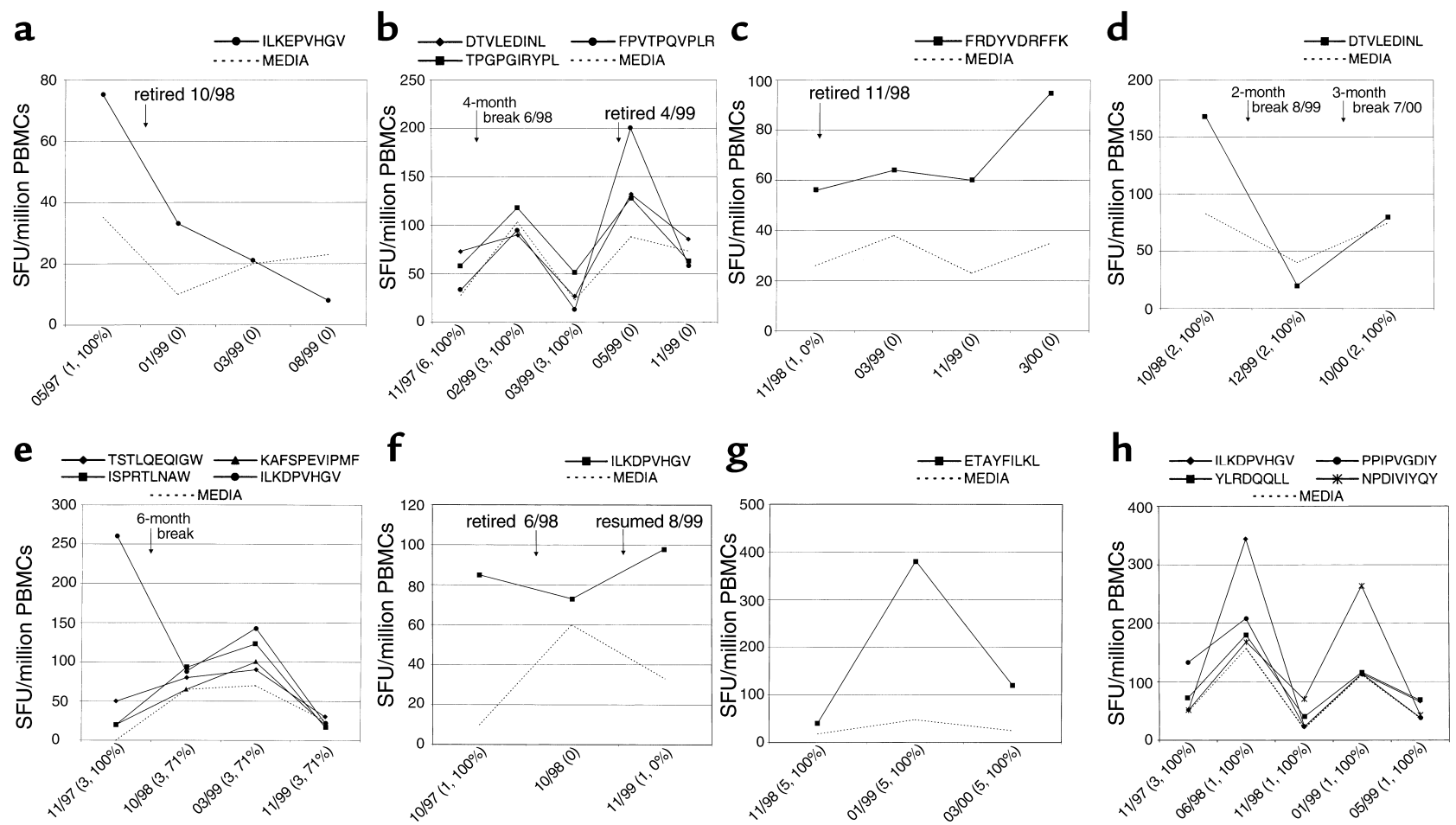

Figure 2

Association between HIV-1 epitope-specific ELISPOT responses (see text for definition of an HIV-1 epitope-specific response) and levels of sex work in persistently HIV-1-seronegative Kenyan sex workers. HIV-1-specific ELISPOT responses are shown on the $y$ axis as SFU/10 $\mathrm{PBMCs}$. Solid lines represent IFN- $\gamma$ release in response to HIV-1 CTL peptide epitopes, and the dashed lines represent the IFN- $\gamma$ response to R10 medium alone. Assay date is shown on the $x$ axis, together with the reported number of daily clients and percentage of condom use. Stopping sex work completely was generally associated with loss of HIV-1-specific ELISPOT responses, demonstrated by ML 1192 (a) and ML 851 (b), although ML 1732 maintained a response for over a year after stopping sex work (c). A temporary break ( $\geq 2$ months) from sex work was also associated with the loss of HIV-1-specific responses (ML 1437) (d), and responses were detected again 1-12 months after resuming sex work in ML 1250 (e), ML 1749 (f), and ML 851 (before retirement) (b). HIV-1-specific responses tended to be maintained in the absence of a break from sex work, shown by ML 1671 (g) and ML 887 (h).

over the course of the study had stopped sex work, while only one of six women who maintained epitopespecific responses had stopped sex work $(\mathrm{OR}=30.0$; $95 \%$ CI, $1.5-612 ; P=0.03)$.

\section{Discussion}

Despite intense HIV-1-infection pressure, a small subgroup of sex workers in Nairobi, Kenya, have remained uninfected for periods of up to 15 years (25). This HIV-1 resistance has been associated with various HIV1-specific immune responses including T-helper responses, plasma and mucosal IgA, and CTL epitope responses, suggesting that resistance may be immune mediated (35). However, the phenomenon of HIV-1 resistance is not absolute: although the incidence of new HIV-1 infections declines sharply after 2 years of follow-up, occasional seroconversions are seen as late as ten years after enrollment, as demonstrated by an update of the survival analysis described previously (25) (Figure 3). In this study we have described all 11 sex workers who had met HIV-1-resistance criteria but subsequently seroconverted during the period January 1, 1996 to December 31, 1999. These women had been followed for an average of 6.9 years before seroconver- sion, had had an estimated 527 unprotected exposures to HIV-1 (based on mean client numbers and condom use, assuming a $17.7 \%$ HIV-1 prevalence among clients; ref. 36), and are quite representative of the overall HIV1-resistant cohort. By definition, seroconversion in this resistant cohort is a rare event and as a result the number of study subjects is necessarily small.

If HIV-1-specific CTL are responsible for resistance to HIV-1 infection, then the most obvious explanation for late seroconversion is that these women did not have preexisting CTL. However, preseroconversion HIV-1 epitope-specific responses were found in four of six (67\%) seroconverters as long as 3 years before conversion, in keeping with the proportion of the cohort demonstrating responses in other studies (refs. 18 and 28; R. Kaul, unpublished observation). These responses were demonstrated by IFN- $\gamma$ ELISPOT in four of four cases and were confirmed in a classic chromiumrelease assay in two cases. It is therefore clear that late seroconversion can occur despite preexisting HIV-specific CTL. Although preseroconversion responses could be tested in only 6 of 11 seroconverters, predicted levels of HIV-1 exposure in these cases did not differ from those without available PBMCs. Ultimately, whether 
Table 4

HIV-1 CTL epitope-specific responses among 22 highly exposed, persistently seronegative sex worker controls

\begin{tabular}{|c|c|c|c|}
\hline Subject & $\begin{array}{l}\text { Epitopes recognized } \\
\text { in IFN- } \gamma \text { ELISPOT }\end{array}$ & $\begin{array}{l}\text { Proportion of } \\
\text { assays positive }\end{array}$ & $\begin{array}{c}\text { Peak response } \\
\text { (SFU } 10^{6} \text { PBMCs) }\end{array}$ \\
\hline \multirow[t]{3}{*}{ ML 851} & DTVLEDINL & $2 / 5$ & 45 \\
\hline & TPGPGIRYPL & $3 / 5$ & 40 \\
\hline & FPVTPQVPLR & $1 / 5$ & 112 \\
\hline \multirow[t]{4}{*}{ ML 887} & ILKDPVHGV & $2 / 5$ & 188 \\
\hline & YLRDQQLL & $4 / 5$ & 30 \\
\hline & PPIPVGDIY & $2 / 5$ & 83 \\
\hline & NPDIVIYQY & $2 / 5$ & 151 \\
\hline ML 1192 & ILKD/EPVHGV & $2 / 4$ & 40 \\
\hline \multirow[t]{4}{*}{ ML 1250} & TSTLQEQIGW & $1 / 4$ & 50 \\
\hline & ISPRTLNAW & $1 / 4$ & 20 \\
\hline & KAFSPEVIPMF & $1 / 4$ & 20 \\
\hline & ILKDPVHGV & $2 / 4$ & 260 \\
\hline ML 1437 & DTVLEDINL & $1 / 2$ & 85 \\
\hline ML 1441 & none & $0 / 1$ & \\
\hline ML 1573 & KYRLKHLWW & $1 / 1$ & 50 \\
\hline ML 1589 & LSPRTLNAW & $2 / 4$ & 50 \\
\hline ML 1593 & none & $0 / 1$ & \\
\hline ML 1601 & DTVLEDINL & $2 / 2$ & 128 \\
\hline ML 1643 & none & $0 / 2$ & \\
\hline \multirow[t]{2}{*}{ ML 1668} & AIFQSSMTK & $1 / 2$ & 40 \\
\hline & YPLTFGWCY/F & $1 / 2$ & 40 \\
\hline ML 1671 & ETAYFILKL & $2 / 2$ & 333 \\
\hline ML 1693 & LSPRTLNAW & $1 / 3$ & 20 \\
\hline ML 1700 & none & $0 / 3$ & \\
\hline ML 1705 & $\mathrm{~N} / \mathrm{A}$ & & \\
\hline ML 1726 & none & $0 / 3$ & \\
\hline ML 1732 & FRDYVDFFK & $2 / 3$ & 37 \\
\hline ML 1747 & none & $0 / 2$ & \\
\hline \multirow[t]{2}{*}{ ML 1749} & ILKD/EPVHGV & $2 / 3$ & 75 \\
\hline & ALKHRAYEL & $1 / 3$ & 25 \\
\hline ML 1766 & none & $0 / 2$ & \\
\hline \multirow[t]{2}{*}{ ML 1792} & DLNMMLNIV & $2 / 4$ & 23 \\
\hline & RAEQASQEV & $2 / 4$ & 308 \\
\hline
\end{tabular}

${ }^{A}$ See text for definition of an HIV-1 epitope-specific response.

preexisting CTL were present in 4 of 6 or 4 of 11 late seroconverters, the fact remains that seroconversion occurred despite HIV-specific CTL in a substantial proportion of these women.

Although amino acid substitutions within immunodominant CTL epitopes can lead to viral escape and disease progression in HIV-infected subjects (22-24), viral epitope sequences from seroconverters matched those recognized by preseroconversion ELISPOT/CTL assays for five of six epitopes. Infection by a potential escape variant occurred for one in six epitopes (ML 857 HPDIVIYQY $\rightarrow$ NPEIIIYQY), and a bulk CTL assay confirmed that preseroconversion PBMCs did not recognize the epitope variant. However, no sequence variation was seen within a second CTL epitope recognized by this subject before seroconversion, which therefore cannot be explained entirely by CTL escape. Furthermore, it is possible that this sequence variation was due to chance, since the amino acid substitutions seen within this epitope have all been described previously in clade A HIV-1 (34). Overall, it seems unlikely that viral CTL escape was a major factor in late HIV-1 seroconversion in these women.

Since HIV-1 epitope-specific responses had been demonstrated before seroconversion, we initially hypothesized that seroconversion might have occurred due to some behavioral factor associated with increased viral exposure, such as an increase in the number of clients or a new type of high-risk sex, that had overwhelmed preexisting protective CTL responses. We performed a case-control study comparing seroconverters with enrollment-matched, persistently seronegative controls. No increase in classical HIV-1 risk-taking behaviors was seen. In fact, although the sample size was necessarily small, cases were more likely than controls to have reduced sex work (and presumably their HIV-1 exposure) over the past year, either by stopping sex work temporarily or by reducing their number of clients.

The association of HIV-1 seroconversion with reduced sex work seems initially counterintuitive, but it might be compatible with a loss or diminution of HIV-1-specific CTL in the absence of ongoing antigenic stimulation, as is seen in chronic HIV-1 infection in the context of complete viral suppression with antiretroviral therapy $(5,6,9,10)$. Antigenic stimulus is required to maintain effector CTL (7). Even memory CTL, which are able to undergo multiple divisions and replenish the effector population (11) and which may be maintained in chronic HIV-1 infection in the absence of detectable virus (6), require persistence of antigen for maintenance in the periphery (20).

Ideally, to test the hypothesis that late seroconversion in these sex workers was related to the loss or waning of HIV-1 epitope-specific responses after a break from sex work, seroconverters should have been assayed immediately after the break. The demonstration that preexisting HIV-1-specific responses had been lost, with subsequent seroconversion upon resuming sex work, would have provided the strongest possible evidence for the hypothesis. Unfortunately, women generally did not go to the clinic until several months after resuming sex work and subsequently seroconverting,

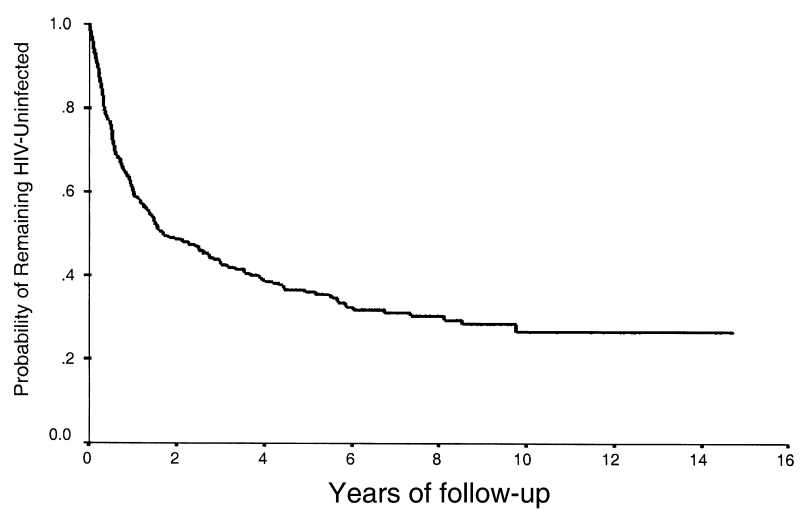

Figure 3

Time to HIV-1 seroconversion after enrollment in the Pumwani sex worker cohort, 1983-1999. 
so this was not possible. However, persistently seronegative women who had not reduced sex work were able to maintain epitope-specific responses, while responses were consistently lost in the context of stopping sex work. These observations suggest that HIV-1 epitope-specific responses would also have been lost following a break from sex work in those women who subsequently seroconverted. However, more frequent testing of sex workers before and after any break from sex work will be necessary to clearly demonstrate an association between reduced antigen exposure, the loss of HIV-specific CTL, and subsequent seroconversion. The timing of HIV-1 testing does not enable us to completely rule out that some women seroconverted before the break from sex work. However, no seroconverter had stopped sex work due to illness, and 4 of 11 women described flulike symptoms upon resumption of sex work, which could have been compatible with acute HIV-1 infection.

The ELISPOT assay used in this study measures IFN- $\gamma$ release by $\mathrm{CD}^{+} \mathrm{T}$ cells in response to specific HIV-1 CTL epitope peptides as a surrogate for conventional chromium-release CTL assays $(19,37)$. The use of MHC-peptide tetrameric complexes to study this HEPS cohort is limited by the fact that precursor frequencies are generally below the threshold of tetramer detection (200/106 PBMCs; ref. 19), and are frequently directed against epitopes that are not available in MHC-peptide tetrameric complexes. In the ELISPOT assay PBMCs are exposed to antigen for a relatively short time (6-16 hours), and so the assay should detect circulating antigen-specific effector (rather than memory) CTL. We did not study memory CTL in the context of stopping sex work. However, it is not clear that a memory CTL population would be able to protect against an infectious mucosal exposure, since for other mucosal infections an effector CTL response may be essential for protection against subsequent challenge (38). It is therefore plausible that CTL-mediated protection against HIV-1 may be lost as effector CTL frequencies fell below an as yet unknown protective threshold.

It is not clear why, after stopping sex work for several months, restarting sex work should result in seroconversion in some women and in the return of HIV-1 epitope-specific responses in others, presumably due to the boosting of memory CTL after antigen exposure. There are several possible explanations. CD8 ${ }^{+}$-lymphocyte responses may persist in some women after a break from sex work, but below the level of ELISPOT detection. Alternatively, epitope-specific responses in blood may not accurately reflect those at the level of the genital mucosa, which may be more relevant in protection from sexual transmission (28). Finally, the influence of antigen exposure on other HIV-1-specific immune responses described previously in this cohort, such as T-helper responses $(13,29)$ and HIV-1-specific IgA (29), could not be examined in this study (although mucosal IgA had been tested before seroconversion in two cases, ML 1203 and ML 1575, and had been detected in one of two; ref. 29). The exact nature of the viral contact after a period of reduced HIV-1 exposure, including such factors as viral phenotype and the dose or route of exposure, may be crucial in determining whether exposure results in boosting of protective immunity or the establishment of productive infection.

In summary, it is clear that HIV-1 resistance is not absolute and that seroconversion can infrequently occur despite preexisting HIV-1-specific $\mathrm{CD}^{+}{ }^{+}$responses. The association between late seroconversion and reduced sex work and the finding that HIV-1-specific effector responses fluctuate depending on levels of antigen exposure, suggests that ongoing antigenic exposure may be necessary to maintain a protective CTL response. The positive implication for preventive HIV-1 vaccine development is that HIV-1 resistance may not be an all-or-none phenomenon, but rather an immunologic state that is inducible given the correct antigenic stimulus. However, it also suggests that maintenance of HIV-1 immune resistance will require ongoing antigenic priming, either through intermittent vaccine boosters or through the use of vaccine strategies employing persistent antigen.

\section{Acknowledgments}

We thank Jane Kamene for assistance in subject recruitment; Kati di Gleria for peptide synthesis; and the women of the Pumwani cohort for their cooperation and enthusiasm. This work was supported by the UK Medical Research Council (S.L. Rowland-Jones, UK Medical Research Council Senior Fellow); the Elizabeth Glaser Pediatric AIDS Foundation (S.L. Rowland-Jones, Elizabeth Glaser Scientist of the Pediatric AIDS Foundation); the Medical Research Council of Canada (MRC) (F.A. Plummer, GR13301; F.A. Plummer, MRC Senior Scientist; R. Kaul, MRC Fellow); and the Rockefeller Foundation (F.A. Plummer, RF 96034).

1. Clerici, M., and Shearer, G.M. 1996. Correlates of protection in HIV infection and the progression of HIV infection to AIDS. Immunol. Lett. 51:69-73.

2. Haynes, B.F., Pantaleo, G., and Fauci, A.S. 1996. Toward an understanding of the correlates of protective immunity to HIV infection. Science. 271:324-328.

3. Rowland-Jones, S.L., and McMichael, A. 1995. Immune responses in HIVexposed seronegatives: have they repelled the virus? Curr. Opin. Immunol. 7:448-455.

4. Letvin, N.L. 1998. Progress in the development of an HIV-1 vaccine. Science. 280:1875-1880.

5. Ogg, G.S., et al. 1999. Decay kinetics of human immunodeficiency virusspecific effector cytotoxic $T$ lymphocytes after combination antiretroviral therapy. J. Virol. 73:797-800.

6. Nixon, D.F., et al. 1999. Molecular tracking of an Human Immunodeficiency Virus nef specific cytotoxic T-cell clone shows persistence of clonespecific T-cell receptor DNA but not mRNA following early combination antiretroviral therapy. Immunol. Lett. 66:219-228.

7. Dalod, M., et al. 1998. Evolution of cytotoxic T lymphocyte responses to human immunodeficiency virus type 1 in patients with symptomatic primary infection receiving antiretroviral triple therapy. J. Infect. Dis. 178:61-69.

8. Ogg, G.S., et al. 1998. Quantitation of HIV-1-specific cytotoxic T lymphocytes and plasma load of viral RNA. Science. 279:2103-2106.

9. Kalams, S.A., et al. 1999. Levels of human immunodeficiency virus type 1-specific cytotoxic T-lymphocyte effector and memory responses decline after suppression of viremia with highly active antiretroviral therapy. $J$. Virol. 73:6721-6728

10. Gray, C.M., et al. 1999. Frequency of class I HLA-restricted anti-HIV CD8+ 
$\mathrm{T}$ cells in individuals receiving highly active antiretroviral therapy (HAART). J. Immunol. 162:1780-1788.

11. McMichael, A.J., et al. 2000. Memory CD8+ T cells in HIV infection. Philos. Trans. R. Soc. Lond. B Biol. Sci. 355:363-367.

12. Pinto, L.A., et al. 1995. ENV-specific cytotoxic T lymphocyte responses in HIV seronegative health care workers occupationally exposed to HIV-contaminated body fluids. J. Clin. Invest. 96:867-876.

13. Fowke, K.R., et al. 2000. HIV-1 specific cellular immune responses among HIV-1-resistant sex workers. Immunol. Cell Biol. 78:586-595.

14. Bernard, N.F., Yannakis, C.M., Lee, J.S., and Tsoukas, C.M. 1999. Human immunodeficiency virus (HIV)-specific cytotoxic T lymphocyte activity in HIV-exposed seronegative persons. J. Infect. Dis. 179:538-547.

15. Goh, W.C., et al. 1999. Protection against human immunodeficiency virus type 1 infection in persons with repeated exposure: evidence for T cell immunity in the absence of inherited CCR5 coreceptor defects. J. Infect. Dis. 179:548-557.

16. Langlade-Demoyen, P., Ngo-Giang-Huong, N., Ferchal, F., and Oksenhendler, E. 1994. Human immunodeficiency virus (HIV) nef-specific cytotoxic T lymphocytes in noninfected heterosexual contact of HIV-infected patients. J. Clin. Invest. 93:1293-1297.

17. Rowland-Jones, S., et al. 1995. HIV-specific cytotoxic T-cells in HIVexposed but uninfected Gambian women. Nat. Med. 1:59-64.

18. Rowland-Jones, S.L., et al. 1998. Cytotoxic T cell responses to multiple conserved HIV epitopes in HIV-resistant prostitutes in Nairobi. J. Clin. Invest. 102:1758-1765.

19. Kaul, R., and Rowland-Jones, S.L. 1999. Methods of detection of HIV-specific CTL and their role in protection against HIV infection. HIV Molecular Immunology Database: Theoretical Biology and Biophysics. B.T.M. Korber et al., editors. Los Alamos National Laboratory. Los Alamos, New Mexico, USA. pp. IV27-IV36.

20. Kundig, T.M., et al. 1996. On the role of antigen in maintaining cytotoxic T-cell memory. Proc. Natl. Acad. Sci. USA. 93:9716-9723.

21. Mortara, L., et al. 1998. Selection of virus variants and emergence of virus escape mutants after immunization with an epitope vaccine. J. Virol. 72:1403-1410

22. Borrow, P., et al. 1997. Antiviral pressure exerted by HIV-1-specific cytotoxic T lymphocytes (CTLs) during primary infection demonstrated by rapid selection of CTL escape virus. Nat. Med. 3:205-211.

23. McMichael, A.J., and Phillips, R.E. 1997. Escape of human immunodeficiency virus from immune control. Annu. Rev. Immunol. 15:271-296.

24. Price, D.A., et al. 1997. Positive selection of HIV-1 cytotoxic T lymphocyte escape variants during primary infection. Proc. Natl. Acad. Sci. USA. 94:1890-1895
25. Fowke, K.R., et al. 1996. Resistance to HIV-1 infection among persistently seronegative prostitutes in Nairobi, Kenya. Lancet. 348:1347-1351.

26. Fowke, K.R., et al. 1998. HIV type 1 resistance in Kenyan sex workers is not associated with altered cellular susceptibility to HIV type 1 infection or enhanced beta-chemokine production. AIDS Res. Hum. Retroviruses. 14:1521-1530.

27. MacDonald, K.S., et al. 2000. Influence of HLA supertypes on susceptibility and resistance to human immunodeficiency virus type 1 infection. J. Infect. Dis. 181:1581-1589.

28. Kaul, R., et al. 2000. HIV-1 specific mucosal CD8+ lymphocyte responses in the cervix of HIV-1 resistant prostitutes in Nairobi. J. Immunol. 164:1602-1611.

29. Kaul, R., et al. 1999. HIV-1-specific mucosal IgA in a cohort of HIV-1resistant Kenyan sex workers. AIDS. 13:23-29.

30. Ngugi, E.N., et al. 1988. Prevention of transmission of human immunodeficiency virus in Africa: effectiveness of condom promotion and health education among prostitutes. Lancet. 2:887-890.

31. Simonsen, J.N., et al. 1990. HIV infection among lower socioeconomic strata prostitutes in Nairobi. AIDS. 4:139-144.

32. Dawood, M.R., Allan, R., Fowke, K., Embree, J., and Hammond, G.W. 1992. Development of oligonucleotide primers and probes against structural and regulatory genes of human immunodeficiency virus type 1 (HIV-1) and their use for amplification of HIV-1 provirus by using polymerase chain reaction. J. Clin. Microbiol. 30:2279-2283.

33. Bunce, M., et al. 1995. Phototyping: comprehensive DNA typing for HLAA, B, C, DRB1, DRB3, DRB4, DRB5 \& DQB1 by PCR with 144 primer mixes utilizing sequence-specific primers (PCR-SSP). Tissue Antigens. 46:355-367.

34. 1999. HIV CTL epitope alignments. HIV Molecular Immunology Database: Theoretical Biology and Biophysics. B.T.M. Korber et al., editors. Los Alamos National Laboratory. Los Alamos, New Mexico, USA. p. I-D-49.

35. Plummer, F.A., Ball, T.B., Kimani, J., and Fowke, K.R. 1999. Resistance to HIV-1 infection among highly exposed sex workers in Nairobi: what mediates protection and why does it develop? Immunol. Lett. 66:27-34.

36. Martin, H.L., Jr., et al. 1994. Preparation for AIDS vaccine evaluation in Mombasa, Kenya: establishment of seronegative cohorts of commercial sex workers and trucking company employees. AIDS Res. Hum. Retroviruses. 10(Suppl. 2):S235-S237.

37. Larsson, M., et al. 1999. A recombinant vaccinia virus based ELISPOT assay detects high frequencies of Pol-specific CD8 T cells in HIV-1-positive individuals. AIDS. 13:767-777.

38. Ahmed, R., and Gray, D. 1996. Immunologic memory and protective immunity: understanding their relation. Science. 272:54-60. 